only disrupted when I realised that my experience of illness was quite common to other persons with a RMD. I can still remember my fascination, in a Patient Reported Outcomes workshop, by discovering that our experience as patients had a bigger relevance that the one we usually conceive.

Then, I felt the need of understanding the research process and specific jargon, so I could relate them with my knowledge on living with a RMD. Otherwise, any insight on the patient perspective would be "lost in translation", due to an inability to establish a correspondence between the scientific terminology and mindset regarding the disease, and the experience of illness and sickness by the individuals. Training opportunities were also recognised as desirable by the above mentioned EULAR recommendations, to increase expertise and understanding of research methods and to promote the patients' self-confidence on their contribution to research.

The Patient Research Partners (PRP) training by EULAR, in 2013, was the first step towards a better understanding of what could be the role of patients and on how to provide a meaningful input from the patients' perspective into research processes. It was followed in 2014 by the training provided by European Patients' Academy on Therapeutic Innovation (EUPATI) for Patient Expert on the Medicines Research and Development Process. This expert-level training was organized in a mixture of independent e-learning coursework and face-to-face training events over a 14-month period. The syllabus involved six modules: Discovery of Medicines \& Planning of Medicine Development, Non-Clinical Testing and Pharmaceutical Development, Exploratory and Confirmatory Clinical Development, Clinical Trials, Regulatory Affairs, Medicinal Product Safety, Pharmacovigilance and Pharmacoepidemiology, HTA principles and practices. Additionally, in 2016, I have attended the 1st EULAR course on Health Economics in Rheumatology.

In the meantime, my background as an anthropologist led me to become interested in Medical Anthropology. Between 2007-2015 my academic research (MA Phil. and PhD) was oriented towards a specialization in Anthropology of Health, with a special interest in Anthropology of Pharmaceuticals.

Based on my personal experience, on this lecture I will focus on the challenges of the role of the PRP trying to fill the gap between the mindsets and practices of different stakeholders.

Navigating through different meanings of symptoms and treatments, the educated patient representative must act like a translator, decodifying the biologic impact of the disease and intervention over the experience of illness on the everyday aspects of living with a RMD. The biggest expectation and challenge might be to bring these aspects forward, as relevant for the other stakeholders, since they shape individual values and patient's preferences.

Although recognised as having a pivotal role, patient's involvement in research may be limited by tokenism or by ineffective patients' participation. Patients' involvement is now a requirement and an added value to any project. But, is the project team ready and willing to listen to patients? Are PRP duly involved in the project, or are they just expected to be recipients without any input of their perspectives into the development and implementation of the research?

The knowledge and education acquired to perform our task enables us to understand science enough to communicate the patient experience in a meaningful way, improving the research. Our added value is, undoubtedly, our experience with the disease, our understanding of the individual values and preferences shaped by the everyday aspects of living with a RMD. We should be taken more seriously, for the benefit of science and patients.

Disclosure of Interest: None declared

DOI: 10.1136/annrheumdis-2017-eular.7243

\section{SP0052 BUILDING PATIENT PARTNERSHIP IN HEALTH CARE SERVICE DESIGN AND DELIVERY}

\section{H. Lempp. Rheumatology, Kings College London, London, United Kingdom}

This paper will present details of the approach to patient and public involvement in health service delivery, health service research and health care education in England. The presentation will be based upon a Logical Framework with the following key elements: inputs, processes, outputs, and outcomes/impacts. Key barrier acting to minimise the impact of building patient partnerships will be discussed, illustrated by examples from our experience in the preparatory stages for our departmental strategy to formalise close Patient Partnership for our research portfolio: (i) establish honorary contracts for patients for the academic Institution and local Hospital Trust; (ii) include patients on the interview panels to appoint project researchers (iii) build in a separate funding within the overall project budget for the costs associated with patient partnership and (iv) manage patient expectations of rapid implementation of the results of research after the completion of projects. Patient Partnership is essential and feasible to make health care service and design relevant.

\section{References:}

[1] Otto AK, Novielli K, Morahan PS.(2006) Implementing the logic model for measuring the value of faculty affairs activities. Acad Med. 2006 Mar;81(3):280-5.

[2] Mockford C, Staniszewska S, Grifiths F, Herron-Marx S. The impact of patient and public involvement on UK NHS health care: a systematic review. International Journal for Quality in Health Care, 2012; 24:28-38.

Disclosure of Interest: None declared

DOI: 10.1136/annrheumdis-2017-eular.7123

\section{THURSDAY, 15 JUNE 2017 WIN \& HOT session}

\section{SP0053 HOT SESSION: VASCULITIS TREATMENT}

\section{R.A. Luqmani. University of Oxford, Oxford, United Kingdom}

The systemic vasculitides are characterized by inflammation of blood vessels, which if untreated, results in tissue or end organ damage or death. Chapel Hill nomenclature is widely used to define different forms of vasculitis according to the size of the predominantly affected vessels. Other forms of vasculitis are not defined by a predominant vessel size (e.g. Behcet's syndrome). Vasculitis associated with the presence of anti-neutrophil cytoplasm antibody (ANCA), termed AAV, is less common than giant cell arteritis (GCA), but considerable advances have been made in understanding the pathogenesis and evidence based treatment for AAV. AAV is divided into three major forms: granulomatosis with polyangiitis (GPA) (Wegener's granulomatosis), microscopic polyangiitis (MPA) and eosinophilic granulomatosis with polyangiitis (EGPA) (Churg-Strauss syndrome). ANCA are implicated in their pathogenesis but not all patients with AAV are ANCA positive. We will review recent EULAR guidelines on therapy for AAV, based on careful structured clinical evaluation of patients, with stratification according to severity. Cyclophosphamide or rituximab (plus glucocorticoid) is used for severe disease, followed by maintenance with azathioprine (AZA) or methotrexate (MTX), and reducing doses of glucocorticoids; or maintenance rituximab. Additional plasmapheresis is indicated for very severe disease; by contrast for less severe disease, MTX or AZA or mycophenolate (plus glucocorticoids) can be used. The evidence for effectiveness is clear for MPA and GPA. A number of studies are underway to improve our use of these existing agents and to test newer, mechanism based treatments such as inhibition CTLA4lg or of the C5 complement pathway in GPA and MPA. For EGPA with severe manifestations, cyclophosphamide and glucocorticoids are recommended. A trial of mepolizumab (inhibitor of interleukin 5 , a potent driver of eosinophil production) in EGPA has recently been completed. IL-6 inhibition with tocilizumab is a significant advance over glucocorticoid monotherapy in treatment of GCA. Apremilast is effective in treating mucocutaneous manifestations of Behcet's syndrome. Relapse is a common feature of many forms of vasculitis and needs to be monitored by structured clinical evaluation. Monitoring of ANCA titres in AAV or the acute phase response in most forms of vasculitis can be misleading and should not serve as sole guide to therapy in the absence of clinical evidence of active disease. Early survival is over $94 \%$ of patients with most forms of vasculitis. Five year survival is $70-75 \%$ for AAV with current therapy. However, if the condition is more severe disease, especially with significant renal impairment, mortality is worse.

Vasculitis remains a challenge. Whilst mortality has dramatically reduced as a result of effective immunosuppression, relapse and chronic damage are significant problems for all forms of vasculitis. We need a better understanding of how to manage and limit the long term chronic effects of vasculitis and its therapy.

Disclosure of Interest: R. Luqmani Grant/research support from: Arthritis Research UK, GSK, MRC, UCSF/OIF, Canadian Institutes of Health Research, The Vasculitis Foundation; Consultant for: GSK, Medpace, Medlmmune, Roche DOI: 10.1136/annrheumdis-2017-eular.7162

\section{THURSDAY, 15 JUNE 2017 Treat-to-target in axSpA: reality or utopy?}

\section{SP0054 THE CONCEPT OF TREAT-TO-TARGET}

\section{J. Braun. Rheumazentrum Ruhrgebiet, 44649 Herne, Germany}

Many illnesses including most rheumatic diseases have substantial effects on well-being and quality of life, including deterioration of physical and mental function and a reduced life expectancy, since they can cause damage to organs and cells. If healing and regeneration cannot be achieved an impairment of organ function can be expected. In acute diseases this may occur rapidly over hours to days and weeks, while it often takes months to years in chronic diseases. However, if treatment is instituted early enough, organ damage may be prevented or diminished.

Critical for an optimal management of diseases with potentially severe outcomes is to determine the responsible thresholds for, for example, disease activity or to define the maximum level of a surrogate marker at which damage is unlikely to occur and, thus, will not be harmful in the long term. Although the optimal aim of therapy is cure, and appropriate therapy may even normalize life expectancy, many chronic diseases such as hypertension, diabetes, rheumatoid arthritis (RA) and ankylosing spondylitis (AS) have remained without curative therapies in the last decades - even though considerable progress has been made. Thus, a strategic therapeutic approach should aim for prevention of future damage, and maximal improvement of compromised organ function. Therefore, a clearly defined threshold of a validated measure that predicts future harm or no or minimal harm, is a target of critical importance for chronic diseases with potentially severe outcomes. Treat-to-target strategies having been developed to achieve this have widespread implications. They should be routinely followed - as long as the potential harm from treatment is carefully balanced against its benefit. Clearly, if inappropriately managed, the consequences of diabetes and hypertension in 